\title{
Exponential and input-output stability are equivalent for linear time-varying systems
}

\author{
R RAVI $^{1}$ and P P KHARGONEKAR ${ }^{2}$ \\ ${ }^{1}$ Control Systems Laboratory, General Electric Research and Development \\ Center, Schenectady, NY 12301, USA \\ ${ }^{2}$ Electrical Engineering and Computer Science, The University of Michigan, \\ Ann Arbor, MI 48109, USA
}

MS received 28 July 1992

\begin{abstract}
We present a new proof showing that for finite dimensional linear time-varying (FDLTV) systems stabilizable by output feedback, exponential and input-output stability are equivalent. This proof does not involve the notion of a dual system as has been done elsewhere and makes use instead of the existence of coprime factorizations. This result is analogous to that in the linear time-invariant case, but because of the time-varying nature of the matrices involved, is not as obvious.
\end{abstract}

Keywords. Exponential stability; input-output stability; coprime factorization; time-varying systems.

\section{Introduction}

A system can be described in at least two different, but related, ways. It can be described in terms of a set of first-order differential equations, commonly known as a state-space model, or it can be described as an operator mapping an input space into an output space. In this note we investigate how the notion of stability with respect to one description is related to that with respect to the other. As is well known, when dealing with state-space models we define stability in terms of the exponential decay of initial states uniformly with respect to time. On the other hand, the operator theoretic description of stability is in terms of the boundedness of the operator norm. We show here that when the system has a stabilizable and detectable realization, these two concepts are identical. This result applies to a broad class of time-varying systems and complements an analogous result in the time-invariant case.

This paper grew out of a technical note that was written a few years ago (Ravi \& Khargonekar 1989). After we had written the note we found that the same result had already been shown for discrete-time systems in Anderson (1982). Apart from the fact that our proof is for continuous-time systems, we believe that our approach to the problem is substantially different in comparison to that of Anderson (1982). Indeed, we do not rely on the dual system to prove our result and only use the fact that for systems with stabilizable and detectable realizations we can readily construct 
coprime factorizations. More importantly, the definitions of stabilizability and detectability in the present paper and in Anderson (1982) are different.

In $\S 1$ we introduce the reader to some standard notation and a few preliminary lemmas. Section 2 contains the statement and proof of our main theorem.

\section{Preliminaries}

Let $\mathscr{R}$ be the set of real numbers and $\mathscr{R}_{+}$the subset of nonnegative real numbers. Let $\mathscr{R}^{n}$ denote the $n$-dimensional Euclidean space, and let the space of $k \times m$ real valued matrices be denoted by $\mathscr{R}^{k \times m}$. The norm in $\mathscr{R}^{k \times m}$ is the induced operator norm defined as: $\|M\|:=\sup _{x \neq 0}(\|M x\|) /(\|x\|)$. Let $\mathscr{M}\left(\mathscr{R}_{+}\right)$be the space of measurable functions mapping $\mathscr{R}_{+}$to $\mathscr{R}$. Let $\mathscr{L}_{p}\left(\mathscr{R}_{+}\right), 1 \leqslant p<\infty$ be the subspace of $\mathscr{M}\left(\mathscr{R}_{+}\right)$ consisting of all functions having a finite $\mathscr{L}_{p}$-norm which is defined as follows: $\|x\|_{p}:=\left(\int_{0}^{\infty}\|x(t)\|^{p} \mathrm{~d} t^{1 / p}\right.$. If $p=\infty$ then the norm is defined to be $\|x\|_{\infty}:=$ ess $\sup _{t \in \mathscr{P}_{+}}(\|x(t)\|)$. Let $P_{t}: \mathscr{M}\left(\mathscr{R}_{+}\right) \rightarrow \mathscr{M}\left(\mathscr{R}_{+}\right)$be the truncation operator defined as usual; $\left(P_{t} x\right)(\tau)=x(\tau)$ for $\tau \leqslant t ; 0$ otherwise. Then the extended space is defined as $\mathscr{L}_{p . e}\left(\mathscr{R}_{+}\right):=\left\{f \in \mathscr{M}\left(\mathscr{R}_{+}\right): P_{t} f \in \mathscr{L}_{p}\left(\mathscr{R}_{+}\right) \forall t>0\right\}$. Corresponding definitions can be made for $\mathscr{L}_{p}^{k \times m}\left(\mathscr{R}_{+}\right)$and $\mathscr{L}_{p, e}^{k \times m}\left(\mathscr{R}_{+}\right)$. For simplicity of presentation, whenever $k, m$ are obvious from the context we will abbreviate the notation for these spaces to $\mathscr{L}_{p}$ and $\mathscr{L}_{p, e}$, respectively.

Let $\mathscr{X}$ denote the set of all linear operators mapping $\mathscr{L}_{p, e}$ to $\mathscr{L}_{p, e}$ and let $G \in \mathscr{X}$. An operator $G$ is said to be causal if $P_{t} G P_{t}=P_{t} G, \forall t \in \mathscr{R}_{+}$. Let us denote the set of linear, causal operators by $\mathscr{X}_{c}$. We say that $G$ is (finite gain) $\mathscr{L}_{p}$-stable if $\|G\|$ : $\sup _{x \in \mathscr{L}_{2, e}, P_{t} x \neq 0, t \in \mathscr{W}}\left(\left\|P_{t} G x\right\|_{p}\right) /\left(\left\|P_{t} x\right\|_{p}\right)<\infty$. Clearly, in this case $G$ is a bounded operator from $\mathscr{L}_{p}$ to $\mathscr{L}_{p}$.

Having introduced the definition of a linear time-varying (LTV) system as an operator, we proceed now to its description in terms of a state-space model. Consider the following set of equations that describe a state-space realization

$$
\Sigma:=\begin{aligned}
& \dot{x}(t)=A(t) x(t)+B(t) u(t), x(0)=x_{0}, \\
& y(t)=C(t) x(t)+D(t) u(t),
\end{aligned}
$$

where $u(t) \in \mathscr{R}^{m}$ and $y(t) \in \mathscr{R}^{p}$ are the input and the output vectors respectively and $x(t) \in \mathscr{R}^{n}$ is the state vector. The matrices are all bounded functions of time. With $x(0)=0$ the system $\Sigma$ generates an input-output operator $G \in \mathscr{X}_{c}$ such that $y=G u$. By using the variation of constants formula we can write down a formula for the operator $G$,

$$
y(t)=\int_{0}^{t} C(t) \boldsymbol{\Phi}(t, \tau) B(\tau) u(\tau) \mathrm{d} \tau+D(t) u(t),
$$

where $\boldsymbol{\Phi}(t, \tau)$ is the state transition matrix of the homogeneous part of (1). In the standard packed matrix form for representing state-space realizations,

$$
\Sigma:=\left[\begin{array}{l|l}
A & B \\
\hline C & D
\end{array}\right] .
$$

\section{DEFINITION 1.}

The system $\Sigma$ is said to be exponentially stable if $\exists c_{1}, c_{2}>0$ such that (s.t.) $\|\boldsymbol{\Phi}(t, \tau)\| \leqslant$ $c_{1} e^{-C_{2}(t-\tau)}, \forall t \geqslant \tau$. 


\section{DEFINITION 2.}

The system $\Sigma$ is said to be stabilizable (respectively detectable) if there exists a bounded function $K(t)$ (respectively $L(t))$ such that system $\dot{x}(t)=(A-B K)(t) x(t)$ (respectively $\dot{x}(t)=(A-L C)(t) x(t))$ is exponentially stable.

It is our intent here to show that the finite gain stability is equivalent to exponential stability for stabilizable and detectable systems.

We end this section with a few results that will be needed in the proof of our main result.

Lemma 2.1. (Desoer \& Vidyasagar 1975, p. 115) Let $G \in \mathscr{X}_{c}$. Then $G$ is $\mathscr{L}_{p}$-stable for each $1 \leqslant p \leqslant \infty$ iff $G$ is $\mathscr{L}_{1}$-stable and $\mathscr{L}_{\infty}$-stable.

Lemma 2.2. Let $G \in X_{c}$ be the input-output operator corresponding to the system $\Sigma$ as in (1). Let $A, B, C, D$ be bounded functions of time. If $\Sigma$ is exponentially stable then $G$ is $\mathscr{L}_{1}$-stable and $\mathscr{L}_{\infty}$-stable.

Proof. Easy to derive from the definition of exponential stability.

Lemma 2.3. (Brockett 1970, p. 193) Let $\boldsymbol{\Phi}(t, \tau)$ be the state transition matrix of the system $\Sigma$ with $A \in \mathscr{L}_{\infty}$. Then if $\exists T>0$ s.t.

$$
\left\|\Phi\left(t_{0}+T, t_{0}\right)\right\| \leqslant \frac{1}{2} \forall t_{0} \in \mathscr{R}_{+},
$$

the system is exponentially stable.

\section{Main result}

Theorem 3.1. Let $G \in \mathscr{X}_{c}$ be the input-output operator corresponding to the system $\Sigma$

$$
\begin{aligned}
& \dot{x}(t)=A(t) x(t)+B(t) u(t), \\
& y(t)=C(t) x(t)+D(t) u(t) .
\end{aligned}
$$

Let $\Sigma$ be stabilizable and detectable and let $1 \leqslant p \leqslant \infty$. Then

(1) $G$ is $\mathscr{L}_{p}$-stable,

$\Leftrightarrow$

(2) $\Sigma$ is exponentially stable.

Proof. $(2) \Rightarrow(1)$. Follows directly from lemma (2.1) and lemma (2.2).

$(1) \Rightarrow(2)$.

From the definition of stabilizability there exists a bounded function $K$ such that $(A-B K)$ describes an exponentially stable system. Define $v:=u+K x$. Then (4) becomes

$$
\begin{aligned}
& \dot{x}=A_{1} x+B v, \\
& y=C_{1} x+D v, \\
& u=v-K x,
\end{aligned}
$$


where $A_{1}:=(A-B K)$ and $C_{1}:=(C-D K)$. Let $T_{x v}$ denote the operator from $v$ to $x$. As $A_{1}$ is internally stable we have, from lemma (2.2), that $T_{x v}$ is bounded. If $\boldsymbol{\Phi}_{1}(t, \tau)$ is the state transition matrix of the system $\dot{x}=A_{1} x$ we have

$$
\begin{aligned}
& x(t)=\int_{t_{0}}^{t} \Phi_{1}(t, \tau) B(\tau) v(\tau) \mathrm{d} \tau, \\
& y(t)=\int_{t_{0}}^{t} C_{1}(t) \Phi_{1}(t, \tau) B(\tau) v(\tau) \mathrm{d} \tau+D(t) v(t), \\
& u(t)=v(t)-\int_{t_{0}}^{t} K(t) \Phi_{1}(t, \tau) B(\tau) v(\tau) \mathrm{d} \tau .
\end{aligned}
$$

This means

$$
y=N v, \quad u=M v,
$$

where $N$ and $M$ have a state-space realization $\Sigma_{N}$ and $\Sigma_{M}$ given by

$$
\Sigma_{N}:=\left[\begin{array}{c|c}
A_{1} & B \\
\hline C_{1} & D
\end{array}\right], \quad \Sigma_{M}:=\left[\begin{array}{c|c}
A_{1} & B \\
\hline-K & I
\end{array}\right] .
$$

From the hypothesis, if $M v \in \mathscr{L}_{p}$ then $N v \in \mathscr{L}_{p}$. Now let us define two systems $X$ and $Y$ in terms of their realizations $\Sigma_{X}$ and $\Sigma_{Y}$ as

$$
\Sigma_{X}:=\left[\begin{array}{c|c}
A-L C & L \\
\hline K & 0
\end{array}\right], \quad \Sigma_{Y}:=\left[\begin{array}{c|c}
A-L C & B-L D \\
\hline K & I
\end{array}\right] .
$$

From the assumption of detectability there is an $L$ such that $A-L C$ is exponentially stable hence both $X$ and $Y$ are exponentially stable. By using simple algebra we can show that

$$
X N+Y M=I .
$$

Multiplying by $M^{-1}$ on the right,

$$
\begin{aligned}
& X N M^{-1}+Y=M^{-1} \\
& \quad \Rightarrow\left\|M^{-1}\right\| \leqslant\|X\|\|G\|+\|Y\| \\
& \quad \Rightarrow\|v\|_{p} \leqslant(\|X\|\|G\|+\|Y\|)\|u\|_{p} \quad \text { (from (7)) } \\
& \quad \Rightarrow\|x\|_{p} \leqslant\left\|T_{x v}\right\|(\|X\|\|G\|+\|Y\|)\|u\|_{p} \quad \text { (from (5)). }
\end{aligned}
$$

Set $\alpha:=\left\|T_{x v}\right\|(\|X\|\|G\|+\|Y\|)$.

Consider now the system $\Sigma_{1}$ described below

$$
\begin{aligned}
& \dot{x}=A x+u, \\
& y=x,
\end{aligned}
$$

and let $G_{1}$ denote the corresponding input-output operator. Clearly $\Sigma$ is exponentially stable iff $\Sigma_{1}$ is exponentially stable. We now define a new system that is equivalent to $G_{1}$ in the input-output sense.

$$
\dot{x}_{1}=(A-B K) x_{1}+u,
$$




$$
\begin{aligned}
& \dot{x}_{2}=A x_{2}+B K x_{1}, \\
& y=x_{1}+x_{2} .
\end{aligned}
$$

Consider first the subsystem described by (12). From lemma (2.2) as $\dot{x}=(A-B K) x$ is exponentially stable we have $x_{1} \in \mathscr{L}_{p}$ whenever $u \in \mathscr{L}_{p}$ and further, $\left\|x_{1}\right\|_{p} \leqslant\left\|T_{x_{1} u}\right\|\|u\|_{p}$. As $K$ is bounded, $x_{1} \in \mathscr{L}_{p} \Rightarrow K x_{1} \in \mathscr{L}_{p}$. Next consider the subsystem described by (13). This is the same as our original system $G$ with $u=K x_{1}$ and hence it immediately follows from the previous arguments that $K x_{1} \in \mathscr{L}_{p} \Rightarrow x_{2} \in \mathscr{L}_{p}$ and also that $\left\|x_{2}\right\|_{p} \leqslant \alpha\|K\|\left\|x_{1}\right\|_{p}$. Coupling this with (11) we have the following chain of implications: $u \in \mathscr{L}_{p} \Rightarrow x_{1} \in \mathscr{L}_{p} \Rightarrow x_{2} \in \mathscr{L}_{p} \Rightarrow y \in \mathscr{L}_{p} \Rightarrow x \in \mathscr{L}_{p}$. Moreover $\left\|x_{p}\right\| \leqslant\left\|x_{1}\right\|_{p}+$ $\left\|x_{2}\right\|_{p} \leqslant\left\|T_{x_{1} u}\right\|(1+\alpha\|K\|)\|u\|_{p}=: M_{1}\|u\|_{p}$.

We have shown above that if $G$ is $\mathscr{L}_{p}$-stable then so is $G_{1}$. All that remains to be shown now is that $\Sigma_{1}$ is exponentially stable or that the states of $\dot{x}(t)=A(t) x(t)$ decay exponentially uniformly with respect to starting times and initial conditions. The case $p=\infty$ can be taken care of right at this juncture. For this particular case the result follows directly from theorem 1, p. 196, in Brockett (1970). For the remainder we consider only the cases $1 \leqslant p<\infty$.

Now let $t_{0}>\varepsilon>0$ be fixed. Clearly the following control function will enable the system (11) to reach $x_{0}$ at time $t_{0}$.

$$
u(\tau):= \begin{cases}\frac{x_{0}}{t_{0}}-A \frac{x_{0} \tau}{t_{0}}, & 0 \leqslant \tau \leqslant t_{0}, \\ 0, & \tau>t_{0} .\end{cases}
$$

Because of the bound on the norm of $A$, it follows that $\|u\|_{p} \leqslant \beta_{2}\left\|x_{0}\right\|_{p}$ for some $\beta_{2}$ and all $t_{0}>\varepsilon>0$ and $x_{0}$. Let $x(t)$ denote the corresponding trajectory for $\Sigma_{1}$. Since $G_{1}$ is $\mathscr{L}_{p}$-stable, there exists $\beta_{1}$ such that $\|x\|_{p} \leqslant \beta_{1}\left\|x_{0}\right\|$. This inequality holds for all $t_{0}>\varepsilon>0$ and $x_{0}$. Next since the norm of $A$ is bounded, we can choose $\beta$ large enough so that $\|x\|_{p} \leqslant \beta\left\|x_{0}\right\|$ holds for all $t_{0} \geqslant 0$ and $x_{0}$.

Finally, to show exponential stability, we need to show that the states actually decay exponentially. To this end we will first get a bound on the $\mathscr{L}_{p}$-norm on the state transition matrix. We will show that such a bound exists and that it is uniform with respect to starting times. This will finally lead us to the exponential bound on the transition matrix. Let $x\left(t_{0}\right)=x_{0}$ be any initial condition for the autonomous system $\dot{x}=A x$. We have, from above, that

$$
\begin{aligned}
x(t) & =\boldsymbol{\Phi}\left(t, t_{0}\right) x_{0}, \\
\Rightarrow \int_{t_{0}}^{t_{1}}\left|x^{\prime}(t) x(t)\right|^{p} \mathrm{~d} t & =\int_{t_{0}}^{t_{t}}\left|x_{0}^{\prime} \boldsymbol{\Phi}^{\prime}\left(t, t_{0}\right) \boldsymbol{\Phi}\left(t, t_{0}\right) x_{0}\right|^{p} \mathrm{~d} t \\
& \leqslant \beta^{p}\left|x_{0}^{\prime} x_{0}\right|^{p} \forall t_{0} \in \mathscr{R}_{+}, \quad \forall t_{1} \geqslant t_{0} .
\end{aligned}
$$

Because in $\mathscr{R}^{n}$ all norms are equivalent, we can replace the euclidean norm in (15) with the $p$ norm. Let $\gamma$ be such that $\|z\|_{p} \leqslant \gamma\|z\| \forall z \in \mathscr{R}^{n}$. Then,

$$
\int_{t_{0}}^{t_{1}} \sum_{i=1}^{n}\left|\left(\Phi\left(t, t_{0}\right) x_{0}\right)_{i}\right|^{p} \mathrm{~d} t \leqslant \gamma \beta^{p}\left|x_{0}^{\prime} x_{0}\right|^{p} \forall t_{0} \in \mathscr{R}_{+}, \quad \forall t_{1} \geqslant t_{0},
$$

where $(\cdot)_{i}$ indicates the $i$ th component of a vector. Let

$$
\Phi\left(t, t_{0}\right)=\left[\Phi_{i, j}\left(t, t_{0}\right)\right]
$$


Define $M_{2}:=n \gamma \beta^{p}$ and choose subsequently $x_{0}=\left[\begin{array}{llll}1 & 0 & \ldots & 0\end{array}\right]^{\prime}, x_{0}=\left[\begin{array}{llll}0 & 1 & \ldots & 0\end{array}\right]^{\prime}$, etc. to get

$$
\int_{\imath_{0}}^{i_{1}} \sum_{i=1}^{n} \sum_{j=1}^{n}\left|\Phi_{i, j}\left(t, t_{0}\right)\right|^{p} \mathrm{~d} t \leqslant M_{2} \forall t_{0} \in \mathscr{R}_{+}, \quad \forall t_{1} \geqslant t_{0} .
$$

Recall that $\boldsymbol{\Phi}\left(t, t_{0}\right)$ is differentiable and that $(\mathrm{d} / \mathrm{d} t) \boldsymbol{\Phi}\left(t, t_{0}\right)=A(t) \boldsymbol{\Phi}\left(t, t_{0}\right)$. As $A \in \mathscr{L}_{\infty}$ we have $\left|(\mathrm{d} / \mathrm{d} t) \boldsymbol{\Phi}_{i, j}\left(t, t_{0}\right)\right| \leqslant n\|\boldsymbol{A}\|\left(\max _{k=1, \ldots, n}\left|\boldsymbol{\Phi}_{k, j}\left(t, t_{0}\right)\right|\right)$. From (17), by interchanging the integral and the summations, we also have that each $\boldsymbol{\Phi}_{i, j}(\cdot, \cdot) \in \mathscr{L}_{p}$. Therefore it follows that $(\mathrm{d} / \mathrm{d} t) \Phi_{i, j}(\cdot,) \in \mathscr{L}_{p}$. For the case $p=1$ exponential stability now follows from Brockett (1970, theorem 3, p. 190). We therefore treat the remaining cases i.e. $1<p<\infty$ next. Using the fact that $\Phi_{i, j}\left(t_{0}, t_{0}\right)=0$, if $i \neq j$, we have the following.

$$
\begin{aligned}
\mid \boldsymbol{\Phi}_{i, j}^{p}\left(t_{1}, t_{0}\right) & =\left|\int_{t_{0}}^{t_{1}} p \boldsymbol{\Phi}_{i, j}^{p-1}\left(t, t_{0}\right)\left(\frac{\mathrm{d}}{\mathrm{d} t} \boldsymbol{\Phi}_{i, j}\left(t, t_{0}\right)\right) \mathrm{d} t\right| \\
& \leqslant p \int_{t_{0}}^{t_{1}}\left|\boldsymbol{\Phi}_{i, j}^{p-1}\left(t, t_{0}\right)\right| \frac{\mathrm{d}}{\mathrm{d} t} \boldsymbol{\Phi}_{i, j}\left(t, t_{0}\right) \mid \mathrm{d} t \\
& \leqslant p\left(\int_{t_{0}}^{t_{1}}\left|\boldsymbol{\Phi}_{i, j}^{p-1}\left(t, t_{0}\right)\right|^{q} \mathrm{~d} t\right)^{1 / q}\left(\int_{t_{0}}^{t_{1}}\left|\frac{\mathrm{d}}{\mathrm{d} t} \boldsymbol{\Phi}_{i, j}^{p-1}\left(t, t_{0}\right)\right|^{p} \mathrm{~d} t\right)^{1 / p} \\
& (\text { Hölder's inequality) } \\
& =p\left(\int_{t_{0}}^{t_{1}}\left|\boldsymbol{\Phi}_{i, j}\left(t, t_{0}\right)\right|^{p} \mathrm{~d} t\right)^{1 / q}\left(\int_{t_{0}}^{t_{1}}\left|\frac{\mathrm{d}}{\mathrm{d} t} \boldsymbol{\Phi}_{i, j}^{p-1}\left(t, t_{6}\right)\right|^{p} \mathrm{~d} t\right)^{1 / p} \\
& (\text { because }(1 / p)+(1 / q)=1) \\
& \leqslant p n\|A\| M_{2}^{p} .
\end{aligned}
$$

If $i=j$ we have $\Phi_{i, j}\left(t_{0}, t_{0}\right)=1$ and we can get the following bound

$$
\left|\boldsymbol{\Phi}_{i, j}^{p}\left(t_{1}, t_{0}\right)\right| \leqslant p n\|A\| M_{2}^{p}+1 .
$$

At any rate we have established that $\boldsymbol{\Phi}_{i, j}\left(t_{1}, t_{0}\right)$ is uniformly bounded $\forall t_{0} \in \mathscr{R}_{+}$, $\forall t_{1} \geqslant t_{0}$, hence it follows that $\left\|\Phi\left(\cdot, t_{0}\right)\right\| \in \mathscr{L}_{\infty}, \forall t_{0} \in \mathscr{R}_{+}$. Note that from that $\left\|\boldsymbol{\Phi}\left(\cdot, t_{0}\right)\right\| \in \mathscr{L}_{p}, \forall t_{0} \in \mathscr{R}_{+}$. Also the bounds on these two norms do not depend on $t_{0}$. In the sequel, let $\|\Phi(\cdot, \cdot)\|_{\infty}:=M_{3}$ and $\|\Phi(\cdot, \cdot)\|_{p}:=M_{4}$. These two facts together with lemma (2.3) will now be used to show that an exponential bound exists for $\boldsymbol{\Phi}\left(t_{1}, t_{0}\right)$. The proof is similar to the one in Brockett (1970). that

To begin with, we use the semigroup property of the state transition matrix to see

$$
\begin{aligned}
\int_{t_{0}}^{t_{1}}\left\|\boldsymbol{\Phi}\left(t_{1}, t_{0}\right)\right\|^{p} \mathrm{~d} \tau & =\int_{t_{0}}^{t_{1}}\left\|\boldsymbol{\Phi}\left(t_{1}, \tau\right) \boldsymbol{\Phi}\left(\tau, t_{0}\right)\right\|^{p} \mathrm{~d} \tau \\
& \leqslant \int_{t_{0}}^{t_{1}}\left\|\boldsymbol{\Phi}\left(t_{1}, \tau\right)\right\|^{p}\left\|\boldsymbol{\Phi}\left(\tau, t_{0}\right)\right\|^{p} \mathrm{~d} \tau \\
& \leqslant M_{3} \int_{t_{0}}^{t_{1}}\left\|\boldsymbol{\Phi}\left(\tau, t_{0}\right)\right\|^{p} \mathrm{~d} \tau \\
& \leqslant M_{3} M_{4}^{p}=: M_{5}^{\nu},
\end{aligned}
$$


from which it follows that

$$
\left(t_{1}-t_{0}\right)^{1 / p}\left\|\mathbf{\Phi}\left(t_{1}, t_{0}\right)\right\| \leqslant M_{5} .
$$

Now define $T:=\left(2 M_{5}\right)^{p}$ to get

$$
\left\|\mathbf{\Phi}\left(t_{0}+T, t_{0}\right)\right\| \leqslant \frac{1}{2}
$$

The conclusion that $G$ is exponentially stable now follows straight from lemma (2.3).

This work was supported in part by the National Science Foundation under grant ECS-8451519, in part by a grant from GE, and in part by US Air Force Office of Scientific Research under grant AFOSR-88-0020. The first author was also supported by a University of Minnesota Graduate Dissertation Fellowship.

\section{References}

Anderson B D O 1982 Internal and external stability of linear time-varying systems. SI AM J Control Optimization 20: 408-413

Brockett R W 1970 Finite dimensional linear systems (New York: John Wiley)

Desoer C A, Vidyasagar M 1975 Feedback systems: Input-output properties (New York: Academic Press)

Ravi R, Khargonekar P P 1989 On the equivalence between exponential stability and inputoutput stability for linear time-varying continuous-time systems, Tech. Report, Department of Electrical Engineering, University of Minnesota, Minneapolis, September 